\title{
Effect of cross-phase modulation on optical phase conjugation in dispersion-shifted fiber
}

\author{
Senfar Wen \\ Department of Electrical Engineering, Chung-Hua Polytechnic Institute, 30 Tung Shiang, Hsinchu, Taiwan, China
}

Sien Chi and Tze-Chung Chang

Institute of Electro-Optical Engineering, National Chiao Tung University, 1001 Ta Hsueh Road, Hsinchu, Taiwan, China

Received January 31, 1994

The temporal effect of optical phase conjugation in dispersion-shifted fiber is studied numerically. Frequency chirping is induced in the conjugate pulse by the signal pulse and the pump wave through cross-phase modulation, which increases with the signal power and may distort the pulse shape when the conjugate pulse propagates in the standard fiber. It is also shown that, when the signal power is low, the optical phase conjugation is nearly ideal.

Recently several experiments showed that chromatic dispersion can be compensated by the optical phase conjugation (OPC) in the fiber. ${ }^{1,2}$ The idea to use the OPC to compensate the dispersion was proposed more than a decade ago. ${ }^{3}$ It has become an important issue because of the introduction of the erbiumdoped fiber amplifier. The operating wavelength of the erbium-doped fiber amplifier is in the 1550-nm band, where the dispersion is high for the standard fiber (STF). To compensate for the fiber loss with the erbium-doped fiber amplifier without sacrificing the bit rate, one needs to overcome the fiber dispersion. The OPC is generated by four-wave mixing in the dispersion-shifted fiber (DSF), in which the pump wavelength is at zero dispersion and the phasematching condition is satisfied. ${ }^{4}$ In this Letter we will solve the wave equation to study the temporal effects in the process and verify whether the conjugator is ideal. It is found that there is frequency chirping in the conjugate pulse that is due to cross-phase modulation (XPM) among the conjugate pulse, signal pulse, and pump wave, which may distort the pulse.

With the carrier $\exp \left[i\left(\beta_{0} z-\omega_{0} t\right)\right]$, where $\omega_{0}$ is the zero-dispersion frequency, the complex envelope of the electric field propagating in the fiber can be written as $^{5}$

$$
i \frac{\partial \phi}{\partial z}-i \frac{1}{6} \beta_{3} \frac{\partial^{3} \phi}{\partial t^{3}}+\gamma|\phi|^{2} \phi=-\frac{1}{2} i \alpha \phi .
$$

Here $\beta_{3}$ represents the third-order dispersion at $\omega_{0}$; $\gamma=n_{2} \omega_{0} / c A_{\text {eff }}$, where $n_{2}$ is the Kerr coefficient and $A_{\text {eff }}$ is the effective fiber cross section; and $\alpha$ is the fiber loss. The term with $\gamma$ in Eq. (1) is usually used to represent self-phase modulation. In fact, it includes all four-wave mixing that is due to the Kerr nonlinearity, for example, XPM and OPC. To show these effects we may decompose the electric-field envelope as

$$
\phi=\phi_{p}+\phi_{s} \exp (-i \Omega t)+\phi_{c} \exp (i \Omega t)
$$

where $\phi_{p}$ is the pump wave with frequency $\omega_{p}=\omega_{0}$ and $\phi_{s}$ and $\phi_{c}$ are the signal wave and the conjugate wave with frequencies $\omega_{s}=\omega_{0}+\Omega$ and $\omega_{c}=\omega_{0}-\Omega$, respectively. Since the slowly varying envelope approximation is assumed in the derivation of Eq. (1), it is required that $|\Omega|<<\omega_{0}$. Substituting Eq. (2) into Eq. (1) and neglecting the high-frequency components, we have

$$
\begin{aligned}
i \frac{\partial \phi_{p}}{\partial z}-i \frac{1}{6} \beta_{3} \frac{\partial^{3} \phi_{p}}{\partial t^{3}} & +\gamma\left(\left|\phi_{p}\right|^{2}+2\left|\phi_{s}\right|^{2}+2\left|\phi_{c}\right|^{2}\right) \phi_{p} \\
& +2 \gamma \phi_{s} \phi_{c} \phi_{p}{ }^{*}=-\frac{1}{2} i \alpha \phi_{p}
\end{aligned}
$$

$$
\begin{aligned}
& i \frac{\partial \phi_{s}}{\partial z}+\frac{1}{6} \beta_{3} \Omega^{3} \phi_{s}+i \frac{1}{2} \beta_{3} \Omega^{2} \frac{\partial \phi_{s}}{\partial t}-\frac{1}{2} \beta_{3} \Omega \frac{\partial^{2} \phi_{s}}{\partial t^{2}} \\
&-i \frac{1}{6} \beta_{3} \frac{\partial^{3} \phi_{s}}{\partial t^{3}}+\gamma\left(\left|\phi_{s}\right|^{2}\right.\left.+2\left|\phi_{c}\right|^{2}+2\left|\phi_{p}\right|^{2}\right) \phi_{s} \\
&+\gamma \phi_{p}{ }^{2} \phi_{c}{ }^{*}=-\frac{1}{2} i \alpha \phi_{s},
\end{aligned}
$$

$$
\begin{aligned}
& i \frac{\partial \phi_{c}}{\partial z}-\frac{1}{6} \beta_{3} \Omega^{3} \phi_{c}+i \frac{1}{2} \beta_{3} \Omega^{2} \frac{\partial \phi_{c}}{\partial t}+\frac{1}{2} \beta_{3} \Omega \frac{\partial^{2} \phi_{c}}{\partial t^{2}} \\
&-i \frac{1}{6} \beta_{3} \frac{\partial^{3} \phi_{c}}{\partial t^{3}}+\gamma\left(\left|\phi_{c}\right|^{2}\right.\left.+2\left|\phi_{s}\right|^{2}+2\left|\phi_{p}\right|^{2}\right) \phi_{c} \\
&+\gamma{\phi_{p}}^{2} \phi_{s}{ }^{*}=-\frac{1}{2} i \alpha \phi_{c}
\end{aligned}
$$

where the terms with the time derivations represent the dispersions of the fields. In Eqs. (3) the terms that represent the XPM can be clearly seen. The last terms in the left-hand sides of Eqs. (3) represent the four-wave mixing that is responsible for the OPC. In our calculations we take $\beta_{3}=7.4 \times 10^{-41} \mathrm{~s}^{3} / \mathrm{m}$, $n_{2}=3.2 \times 10^{-20} \mathrm{~m}^{2} / \mathrm{W}$, and $A_{\text {eff }}=35 \mu \mathrm{m}^{2}$. As an example, we take the propagation of a Gaussian pulse, which is assumed to be

$$
\phi_{1}=\sqrt{P_{1}} \exp \left[-\frac{1}{2}\left(2 t / \tau_{1}\right)^{2}\right],
$$




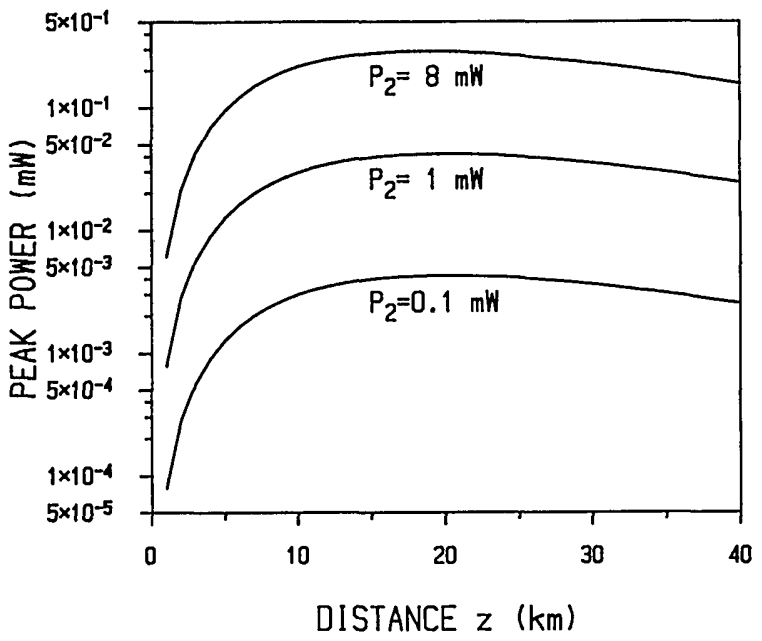

Fig. 1. Peak powers of the conjugate pulses along the DSF for 8-mW pump power and various signal powers $P_{2}$.

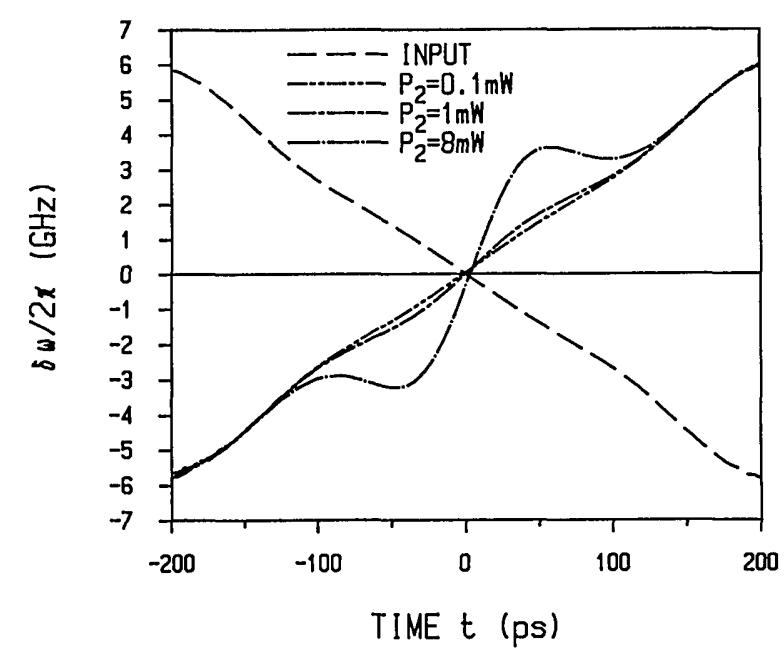

Fig. 2. Instantaneous frequencies $\delta \omega$ of the conjugate pulses after they propagate $20 \mathrm{~km}$ in the DSF for the cases shown in Fig. 1 . The $\delta \omega$ of the input signal pulse is also shown.

where $P_{1}$ and $\tau_{1}$ are its initial peak power and pulse width, respectively. The carrier wavelength $\lambda_{s}=1542.9 \mathrm{~nm}$. The pulse at first propagates in a $80-\mathrm{km}$-long STF with a $0.22-\mathrm{dB} / \mathrm{km}$ loss and $16-\mathrm{ps} /(\mathrm{km} \mathrm{nm})$ dispersion. The Kerr coefficient and effective fiber cross section of the STF are assumed to be the same as the DSF for simplicity. We take $P_{1}=1.43 \mathrm{~mW}$ and $\tau_{1}=100 \mathrm{ps}$. At the end of the fiber the pulse broadens, and its pulse width becomes $116.9 \mathrm{ps}$. The pulse is then amplified to a peak power of $P_{2}$ and is launched into the DSF with a zero-dispersion wavelength at $1546.7 \mathrm{~nm}$ and a $0.24-\mathrm{dB} / \mathrm{km}$ fiber loss. The coupling loss is neglected for simplicity. The pump wave is at the zero-dispersion wavelength and has a power of $P_{p}$. Thus the initial condition used to solve Eq. (1) is

$$
\phi=\sqrt{P_{p}}+\phi_{2} \exp (-i \Omega t),
$$

where the pump wave is a continuous wave, $\phi_{2}$ is the input signal pulse, and $\Omega$ is the frequency deviation from the pump wave. Figure 1 shows the peak power of the conjugate pulse at $\lambda_{c}=1550.5 \mathrm{~nm}$ along the DSF for $P_{p}=8 \mathrm{~mW}$ and various signal powers $P_{2}$. It is seen that the power of the conjugate pulse increases with the signal power and that the optimum length is $\sim 20 \mathrm{~km}$, which agrees well with the experiments. ${ }^{1,2}$ At the optimum length the instantaneous frequencies $\delta \omega$ of the conjugate pulses with different $P_{2}$ values are shown in Fig. 2, for which $\delta \omega=-\partial \psi / \partial t$ and $\psi$ is the phase of the pulse. In Fig. 2 the $\delta \omega$ of the input signal pulse is also shown for comparison. The frequency chirping of the input pulse is due to chromatic dispersion and self-phase modulation in the STF. One can see that the $\delta \omega$ of the conjugate pulse is inverted with respect to the signal pulse. When $P_{2}$ is low $\delta \omega$ is almost equal to the negative $\delta \omega$ of the input pulse. When $P_{2}$ is high there is additional frequency chirping that is due to the XPM from the signal and the pump wave. The pulses broaden only slightly in the DSF because

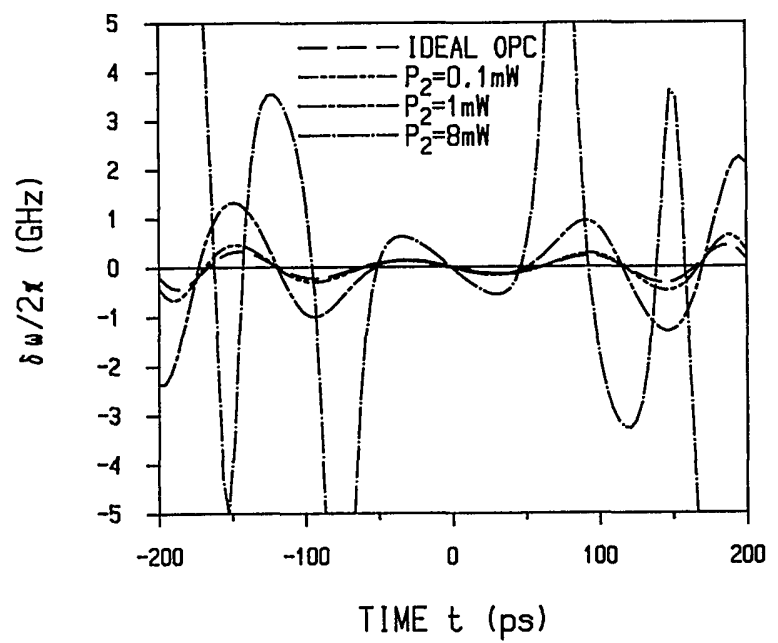

Fig. 3. Instantaneous frequencies $\delta \omega$ of the conjugate pulses after they propagate $80 \mathrm{~km}$ in the STF. The cases obtained from various signal powers $P_{2}$ are shown, as well as the $\delta \omega$ with ideal OPC.

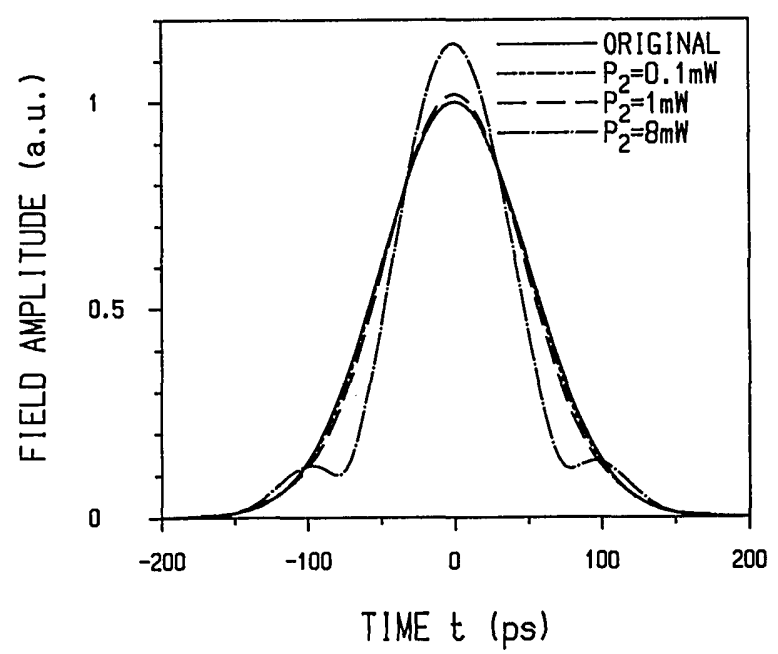

Fig. 4. Corresponding pulse shapes of the conjugate pulses shown in Fig. 3. The original pulse shape given by Eq. (4) is also shown. The amplitudes of the pulses are normalized. 
of low dispersion. After propagating $20 \mathrm{~km}$ in the DSF the pulse widths of the conjugate pulses become $116.8,117.2$, and 120.4 ps for $P_{2}=0.1,1$, and 8 $\mathrm{mW}$, respectively. Unlike the case of the input pulse width, there is almost no further pulse broadening in the DSF when $P_{2}$ is low. The broadening increases with the signal power because of the broadening of the signal pulse, which is enhanced by the frequency chirping that is due to self-phase modulation because its wavelength is in the positive dispersion regime in the DSF. From Eqs. (3b) and (3c) the group velocities of the signal and conjugate pulses are the same. Since there is no walk-off between the two pulses the shape of the conjugate pulse follows the signal pulse. The walk-off between the pump wave and the pulses is only $6.6 \mathrm{ps}$ after the pulses propagate $20 \mathrm{~km}$. Because the walk-off is small there is a dip on the part of the pump wave that overlaps the pulses as a result of the pump depletion, which increases with the signal power. After the pulses propagate $20 \mathrm{~km}$ in the DSF the relative power depth of the dip is $0.32 \%$, $3.17 \%$, and $21.8 \%$ for $P_{2}=0.1,1$, and $8 \mathrm{~mW}$, respectively. This shows that the contribution of the pump wave to the XPM is small compared with the signal pulse in these cases. We take the conjugate pulse at $20 \mathrm{~km}$ as the output pulse, which is then amplified so that its pulse energy is the same as that launched into the first STF and so that it propagates along the second STF. From the theory of OPC the pulse shape of conjugate pulse will be restored when the pulse experiences the same chromatic dispersion that is experienced by the original pulse. Since $\lambda_{s}$ and $\lambda_{c}$ are nearly equal we assume that the dispersion of the second STF at $\lambda_{c}$ is the same as that of the first STF at $\lambda_{s}$, for simplicity. Figures 3 and 4 show the $\delta \omega$ and pulse shapes, respectively, of the conjugate pulses obtained from various $P_{2}$ values after the pulses propagate $80 \mathrm{~km}$. In Fig. 4 the original pulse shape given by Eq. (4) is shown for comparison. Figure 3 also shows the $\delta \omega$ curve that we calculated by assuming ideal OPC, for which the resident frequency chirping is due to the combined effect of the dispersion and self-phase modulation in the STF. The combined effect cannot be compensated completely by the OPC in the lossy medium. ${ }^{6}$ We can reduce such a resident frequency chirping by decreasing the input powers to the STF's. One can see that, for the case with a low $P_{2}, \delta \omega$ is almost the same as the ideal case; i.e., the OPC in the DSF is nearly ideal, and the pulse shape is restored. When $P_{2}$ is high there is significant resident frequency chirping that is due to the XPM from the signal pulse and the pump wave in the DSF. As Fig. 4 shows, the pulse is distorted for such a case. Although its width narrows, the signal pulse will be seriously distorted on further propagation in the STF's, and there will be dispersive waves spreading out, which are not shown here. Therefore for long-distance propagation, with the fiber dispersion periodically compensated by the OPC, it is not desirable to use high signal power to amplify the conjugate pulse.

In conclusion, we have shown frequency chirping of the conjugate pulse that is due to the signal pulse and the pump wave through XPM in the DSF. When the signal power is high the power of the conjugate pulse is amplified, but the frequency chirping is enhanced, which leads to pulse distortion when the conjugate pulse propagates in the STF. It has also been shown that, when the signal power is low, the OPC is nearly ideal.

This study is supported by the National Science Council of China under contract NSC 83-0417-E009013.

\section{References}

1. S. Watanabe, T. Naito, and T. Chikama, IEEE Photon. Technol. Lett. 5, 92 (1993).

2. R. M. Jopson, A. H. Gnauck, and R. M. Derosier, Electron. Lett. 29, 576 (1993).

3. A. Yariv, D. Fekete, and D. M. Pepper, Opt. Lett. 4, 52 (1979).

4. K. Inoue and H. Toba, IEEE Photon. Technol. Lett. 4, 69 (1992).

5. G. P. Agrawal, Nonlinear Fiber Optics, 1st ed. (Academic, Boston, Mass., 1989), p. 40.

6. R. A. Fisher, B. R. Suydam, and D. Yevick, Opt. Lett. 8, 611 (1983). 